\title{
Counselling training in Afghanistan: the long term development of the INSPIRE project
}

Dr Lucia Berdondini (University of East London. UK), Ali Kaveh (University of Herat, Afghanistan), Sandra Grieve (Northern School of Psychodrama, UK)

\begin{abstract}
Between 2010 and 2014, the British Council funded a project under a scheme called INSPIRE, which involved training a group of 20 Afghan practitioners in counselling skills. The participants were from Kabul and Herat and the University of Herat were partners in the project. The ethos of the programme was based on co-constructing a model of transcultural training that could be applicable within the Afghan context (Berdondini et al., 2014). As an outcome, in 2016 the Afghan Ministry of Higher Education approved the launch of a Counselling Department and a Student Counselling Service within the University of Herat. This article aims to present and analyse the long term development of INSPIRE in Afghanistan from the perspective of some participants. Reflections on future implementation of this approach and training programs are also included.
\end{abstract}

Keywords: counselling training, Afghanistan, INSPIRE project, transcultural training

\section{Introduction: the INSPIRE Project (2010-2014)}

Numerous studies report alarmingly high levels of mental health disorders among the Afghan population, including adversity-related distress and non-psychotic disorders (Alemi et al., 2018; Miller at al., 2009; Panter-Brick, et al. 2009). In 2003, 'Mental Health in Afghanistan' became part of a basic package of health services, developed by the Ministry of Public Health. Psychosocial counselling was incorporated in 2010 (National Mental Health Strategy, 2009-2014). Since then a number of intervention programs have been applied to develop mental health training and service resources for the Afghan population (Sayed, 2011). One of the key aspects identified in the National Mental Health Strategy was the creation of a plan of action to overcome cultural barriers and to develop models of treatment compatible with Afghan traditions and belief systems.

Considering the challenges regarding the development of a mental health support system, Babury \& Hayward (2013) highlighted how vital it is to engage the Higher Education Institutions (HEIs). In particular, they stressed the importance of implementing specific counselling training programs within Higher Education to overcome the lack of resources available for the professional 
development of this role and of other helping professions (such as social workers) in the country (Babury \& Hayward, 2013).

In line with the points above, the authors of this paper were awarded in 2010 a grant offered by the British Council, under a scheme called INSPIRE, for a three-year project. The aim of the project was to design a transcultural counselling skills training programme for Afghan practitioners such as doctors, nurses, lecturers, university students and psychosocial counsellors. The institutions involved were the University of Strathclyde (UK) and the University of Herat (Afghanistan). Because of the success of the project and the high demands for services coming from the Province of Kabul, in addition to Herat, further funding was awarded to extended to a fourth year (Berdondini et al., 2014). During the 4-year project a total of 20 participants completed 120 hours of training in counselling skills, while a sub group of 8 participants were involved in 60 hours of training for trainers. This additional course equipped the 8 people with skills and competences to design and facilitate counselling skills courses themselves. The theoretical framework of the whole training project was humanistic and its nature was mainly experiential.

The structure of the course included a variety of inputs:

- Lectures on the theory of Person-Centred Counselling (Rogers, 1951).

- Skills practice sessions (in English and in Dari).

- Activities focused on developing self-awareness and reflectivity.

- Creative therapeutic techniques derived from approaches such as Gestalt therapy (Polster \& Polster, 1973) and Psychodrama (Holmes, et al., 1994) in order to meet different cultural needs and values.

- Supervision sessions (also used as a form of assessment) using Interpersonal Process Recall (IPR, Kagan \& Kagan, 1997).

- Regular revision of the training, through feedback and evaluation forms, in order to modify/adjust/amend the course according to the participants' experience and learning needs across the four years.

Since the beginning of this project, it clearly appeared that both the content and process of the course needed to be negotiated between the two trainers and the trainees. The group of participants was composed of practitioners from different fields (medical doctors, nurses, students and lecturers) and with different needs in regards to integrating counselling skills into their professional role. In the original design of the training, the theories to be discussed in class (mainly 
referring to humanistic therapies such as Person Centred, Gestalt and Psychodrama) were presented and integrated with a strong experiential element, in order to become easily understandable and meaningful to participants. However, this very same element, since day one, had to go through a series of negotiations and creative adjustments within the group, for cultural reasons. For example, one of the first issues encountered was the fact that in the Afghan culture a lot of topics are 'taboo', such as being in love with someone or feeling angry at close family members. These subjects cannot be explicitly discussed between people. Thus, creative methods, culturally meaningful to the group, were introduced to work experientially on self-awareness, skills practice or live demonstrations of therapeutic sessions, such as the use of metaphors, psychodrama, stories and poems, arts, stones and toys (Berdondini, et al, 2014). In this way, the emphasis around developing therapeutic skills was more focused on the 'process' rather than the 'content' and the sessions were often carried out without addressing overtly the actual 'matter'. Participants felt comfortable to work with forms of art and creative tools that are part of their cultural expressiveness. At the same time, during live demonstration between trainers and trainees and skills practice between trainees, the sessions were 'person-centred' in terms of respecting the client's pace and initiative to when and how using arts or objects to explore what they were bringing. The counsellor's role was mainly to facilitate the client's connection with the experience of being in the 'here and now' and of feelings and meanings related to what was happening, without interpreting or trying to find a solution or directing the work. The outcome of this creative transcultural approach proved to be therapeutically very effective, with feedback from participants stressing the 'life changing' power of the sessions and the entire training (Berdondini et al, 2014).

Nevertheless, there were also topics that could be addressed explicitly within the group, such as individual differences with regards to religious believes, lack of safety and security in the country, inner resources and resilience. The students decided the modality of delivery for each of these lectures and as a result, some of them were led by the trainees themselves and included creative writing, analysing extracts from traditional book texts and facilitating group discussions. The nature of the whole course, since the first year, was 'student-centred' and its structure was 'co-constructed' between trainers and trainees, in the sense that participants were engaged as much as the trainers in deciding which contents were more relevant to their learning and which method of delivery was the most appropriate to their cultural values and needs (Proctor, 2008; Berdondini et al., 2014). Each year the training offered was a total of 120 hours, organized in two blocks of 60 hours each. At the end of each block, evaluation forms were filled in and they contributed to adjust and amend the next version of the training course for the following year. The last group, trained during the year 2013-2014 reached $100 \%$ in student satisfaction. When the training was completed, all participants 
were able to carry out counselling sessions within their professional role. Eight selected participants from these training courses in 2013-14 were also trained as trainers.

In consequence of the INSPIRE project, in 2016 the Counselling Department at Herat University was established, after a series of further training and capacity building programs. Some of the lecturers and students from the Psychology Department of Herat University were trained in counselling skills through the INSPIRE project. The Department is still active with about 100 students every year. A Students Counselling Service was also opened in the same University and run by the lecturers trained with INSPIRE.

During the five years post-completion of this project psychosocial counselling training in the country has evolved and in particular, the specific transcultural humanistic approach developed during INSPIRE has been implemented in a number of different contexts, such as Non-Government Organizations, schools, prisons, mental health centres. Currently, there are only two cities that offer counselling training in Higher Education in Afghanistan: Kabul, with two Departments offering courses at BA level and Herat with one. (Afghan Ministry of Higher Education, 2018).

This article aims to explore the evolvement and the impact of the INSPIRE project from the perspective of some of the participants who attended the courses and who are still actively involved in delivering counselling services in Afghanistan. In particular this study was asking the following:

The research question of this study was:

1) How did the implementation of the INSPIRE project evolve?

Sub-questions were:

a) What seems to work particularly well for Afghan clients?

b) What seems to work less for Afghan clients?

In order to respond to these questions a semi-structured self-report form was distributed to some of the former participants. In particular, this report asked them to reflect on the impact that the training had on their personal and professional lives, on other aspects such as employability, and the strengths and limitations of the humanistic approach in relation to the Afghan context. This data were analysed qualitatively using Thematic Analysis (Braun \& Clarke, 2006).

\section{Methodology}

In spring 2018, the Counselling Department of the University of Herat approved the Ethical proposal for the current study and a search was done to contact the 20 participants who were trained in the programme over the four years. It was possible to track back only 15 people from the original 
number of participants (some had left the country). An invitation letter explaining the aim of the study and a consent form were sent to each one of them.

\section{Participants}

A total of 10 participants, 7 men and 3 women, responded to the invitation letter and signed the consent form, accepting to participate in this study. The data were collected electronically via email. At the time of the research all participants were working as counsellors in different contexts in Afghanistan, such as national and international Non-Government Organizations (NGOs), schools, prisons, orphanages, mental health centres in the Province of Kabul and Herat.

\section{Data analysis}

The data were analysed using Thematic Analysis in accordance to the guidelines of Braun and Clarke (2006). Eight of the reports were written in English and two in Dari, translated into English by one of the authors. The process for the data analysis consisted reading the materials several times in order to become familiar with them. Generation of codes followed, with consequent search and review of identified themes, including a second re-coding of data until it produced what the authors considered a satisfactory 'thematic map' (Braun \& Clarke, 2006) (Fig. 1).

\section{Findings}

The final themes that emerged have been organized as following:

\section{A different way of relating to self and to others.}

\section{Impact on professional life;}

Sub-themes:

i. Working experiences after the training.

ii. Approaches used with clients.

3. Applicability of the humanistic approach to Afghan clients.

Sub-themes:

i. Useful aspects

ii. Limitations

iii. Gaps in services

A different way

of relating to self and others

\section{Impact on Professional}

life
Applicability of

the humanistic

approach to

Afghan clients 
Fig. 1. Final themes and sub-themes.

\section{A different way to relate to self and to others}

All participants highlighted how the experience of INSPIRE had impacted their personal development and their perspective on life itself. They seemed to have gained more capacity to look after themselves and to relate to other people in a non-judgmental way.

P1: "It changed my perspective on my life...I became more independent.."

P2: "I always was taking care of my clients and now I got the importance of self-care as well".

P3: "I now accept easily people, make good relation with them and finally it made my life easier in this society".

P4: "After the training I have decided not to judge people and accept them the way they are...I also received good comments from my friends who noticed my changes".

These findings seem to support what was already reported at the end of the training itself through the evaluation forms (Berdondini et al., 2014) were the experience of the programme was described as 'life changing' for most of the participants. From the current reports, it looks like this deep impact on themselves and their relationships with others has been maintained over time, in spite of the difficult environment and the continuous challenges of living in a country like Afghanistan.

\section{Impact on professional life}

Reflections about the impact that the experience of INSPIRE had on the participants' professional life are represented though this theme. In general the reflections seem to claim a stronger clarity about what counselling can and cannot provide and a deeper strength in their own abilities:

P2: "I understood that I can establish a good relationship with clients and care for them"

P3: "I have learnt how to connect with my clients, how to psychologically help them and improve their ability to solve their problems" 
P4: "I have learnt many techniques that helped me having better counselling sessions with various cases, I have learnt how to start a session, how to make the client comfortable and trust you, how to work with people who are not willing to speak".

P9: "It helps me go deeper into the problems with the clients".

From the analysis of the data two sub-themes emerged.

i. Working experiences after the training.

Each participant described their career since the end of the training. Most of them started working with children (some as volunteers) in schools and in orphanages. At the time of the study, participants shared how they saw adult and children clients and worked full time for international Humanitarian Organizations in the role professional counsellors. All of them provided training and 'awareness sessions' for parents and family in the community. Some of them were now specialized in working with vulnerable women, and victims of domestic violence.

Some of them became Lecturers at the University of Herat in the Counselling Department:

P9: "Since the very beginning, I used to work as a teaching assistant and counsellor as well as conducting Person-Centered therapy training for a huge number of Herat University students with other members of this program and I also conducted this training on my own for two separate groups of psychology students".

One participant worked in a female prison. Here are her reflections on this experience and a feedback from one of their clients in the prison:

P3:" It was the first time that such wide program had been offered to them. They believe this is the most useful program that had huge impact on their mental health situations...they are very thankful to this program because now they know how to deal with the tough situation that is the prison. Here is an example of prisoner's comment about program:

I'm very satisfied with you, I learned a lot from you. Because of you my stresses and sorrow reduced. After having counselling sessions my life changed. I will be very happy if you continue working with us. I'm very thankful, thank you so much'."

ii. Approaches used with clients. 
All of them used the Person Centred approach as a foundation to relate with clients and establish a therapeutic alliance. Some of them used other approaches as well, depending on the client's needs, such as CBT, Trauma Sensitive Approach, Psychodrama or creative forms of Gestalt therapy:

P6: "clients can expressed themselves in many ways using different tools, like painting, drawings, toys.... have learnt to use such instruments in my professional life".

P9: "I usually use eclectic approach for my therapy session like Person Centre Therapy, CBT and sometimes Existential approach. I use the approaches according to the different cases I work with" Indeed some of the participants kept studying counselling after the INSPIRE project with postgraduate courses in other institutions abroad, for example in India, and developed an integrative stance with a pluralistic philosophy (Cooper \& McLeod, 2011) where different approaches are used for different clients, depending on what seems more useful and suitable for each one of them.

\section{Applicability of humanistic approach with Afghan clients}

In reflections about their professional experience and the impact that INSPIRE had on their work with clients, participants identified some useful and some less useful aspects.

i. Useful aspects

Some of the aspects that seemed to be particularly useful, refer specifically to the Person-Centred theoretical stance.

P1: "The Person Centred approach gives time to the client to vent their emotions and make decisions by themselves"

P2:"Women are empowered, supported and resourced to claim their rights [...] and to refer them to their internal and external resources"

P3:" Women who suffer from domestic violence are rejected from their family and society. .....] person-centred counselling helps them a lot to build a good trust and relationship with themselves...most of my clients are grateful to be listened to and respected".

P9: "This approach and its techniques are amazing for the process of rapport building or trust building"

ii. Limitations 
At the same time, all participants identified aspects of this approach that do not always seem beneficial for all the clients; for example, when they suffer from "severe mental health issues" (P4) or when they come with a specific question looking for guidance and find that starting a dialogue means the counsellor wanting to interfere with their life:

$P 1$ "Most of them stop speaking and say 'Guide me to solve my problems and don't ask me about my secrets'”.

Disclosing personal matters is a quite challenging issue in the Afghan culture:

P4: "In a close society like Afghanistan it is really difficult for people to trust and say their personal issues to others, even a counsellor. It is because of bad experiences of being judged, people are too much worried about the confidentiality."

In other examples, especially related to women clients, participants highlighted the fact that at times families can get in the way of accessing therapy or represent an insurmountable obstacle:

P2: "Patriarchy and families that do not allow women to go to counselling centres".

P3: "At times it was a big challenge for me because I wanted to help them but their problems was because of others, like husband or family".

iii. Gaps in services.

Finally, some participants identified gaps in the services that are currently offering counselling support in Afghanistan and in the structure that support the professional figure of counsellors themselves. Many issues seem to be related to difficulties in making the counselling services more accessible, but some issues seem also related to the absence of specialised trained counsellors and of supervision.

P2: "Lack of consultants and counselling centres, people lack of trust in counselling, absence of professional and expert counsellors and distance, lack of access to available counselling services"

P3: "One thing is lack of supervision sessions for counselling. When I started my work it was very difficult for me to get out of my clients' problems...it affected me a lot".

\section{Conclusions}

This paper presents some reflections and follow up analysis on how the INSPIRE project that was carried out between 2010 and 2014 in Afghanistan and focused on how developing a transcultural training course in counselling, has impacted on the long term personal and professional development of the participants. 
From the data collected, it seems that at least half of the people originally trained are still practicing as counsellors and some of them even engaged in further Post Graduate training and development. They are working in a variety of different contexts in their country, from mental health centres, to schools, women prisons and orphanages. The INSPIRE project indeed contributed to the creation and implementation of the profession of counselling in Afghanistan and the Counselling Department of the University of Herat is still running BA courses.

Another element that emerges from the data is that the transcultural approach developed during this project is still applied and valued and it is contributing to the wellbeing of vulnerable people in Afghanistan, especially women and children. One quite interesting factor is the explicit recognition from all the participants of some specific benefits of Person-Centred approach for Afghan clients, for example when establishing an initial rapport and as a process of empowerment, particularly for women. This argument is quite controversial, as non-directive counselling has been described as non-suitable for certain cultures (MacDougall, 2002), including the Islamic one (Moracco, 1978). Said that, there have been studies that discuss the power of this approach in the Middle East, when introduced with "insider sensibility" (Al-Thani, \& Moore, 2012). It is important to stress though, that some of the participants of our study also highlighted limitations of this approach, for example when 'severe mental health issues' are involved, or when the clients are illiterate. It was also reported that family and community systems may represent obstacles for individuals wanting to access counselling facilities and it was argued that there is a lack of trust towards the concept of confidentiality. The stigma related to mental health in this culture is still very high and recognised in several studies (Ciftci, et al. 2013; Soheilian \& Inman, 2009). Further research needs to be conducted in order to explore much more in depth the impact of this specific counselling approach on Afghan clients, especially from their direct point of view and experience.

From the data it emerges that additional improvements can be developed for the model of counselling that has been designed within the INSPIRE project. One could be the expansion of its psychosocial/transcultural aspects by creating and introducing other culturally sensitive and meaningful interventions, such as community/family psychosocial programmes that could impact the perception and knowledge about counselling and facilitate the accessibility to the services.

Finally, a crucial aspect, also from an ethical point of view, seems to be the need to introduce in the Afghan counselling system, training in supervision, as it is an essential dimension for the safety of clients and counsellors (Proctor, 2008; Page \& Wosket, 2003) and it is still non-existent, currently. 


\section{References}

Alemi, Q., Stempel, C., Koga, P.M., Montgomery, S., Smith, V., Sandhu, G., Villegas, B. \& Requejo, J. (2018) Risk and protective factors associated with the mental health of young adults in Kabul, Afghanistan, BMC Psychiatry, 18:71, https://doi.org/10.1186/s12888-018-1648-4.

Al-Thani, A. \& Moore J. (2012) Nondirective counseling in Islamic culture in the Middle East explored through the work of one Muslim person-centered counselor in the State of Qatar. Person Centred and Experiential Psychotherapies, 11 (3), 190-204.

Babury, M. O. \& Hayward, F. M. (2013) A Lifetime of Trauma: Mental Health Challenges for Higher Education in a Conflict Environment in Afghanistan, Education Policy Analysis Archives, 21 (68), Retrieved in September 2018 by http://epaa.asu.edu/ojs/article/view/1219.

Berdondini, L. Grieve, S., Kaveh, A. (2014) The INSPIRE project: using the 'unknown' to co-construct a training course on humanistic counselling in Afghanistan, International Journal for the Advancement of Counselling. 36 (3), 305-316.

Braun, V. \& Clarke, V. (2006) Using thematic analysis in psychology, Qualitative Research in Psychology, 3, 77-101.

Ciftci, A., Jones, N., \& Corrigan, P. W. (2013) Mental Health Stigma in the Muslim Community, Journal of Muslim Mental Health, 7 (1), 17-32.

Cooper, M. \& McLeod, J. (2011). Pluralistic Counselling and Psychotherapy. London: Sage.

Egan, G. (1986) The Skilled Helper: A Systematic Approach to Effective Helping (3rd edn), Pacific Grove, Calif.: Brooks/Cole.

Holmes, P. Karp, M and Watson, M (eds) (1994) Psychodrama Since Moreno, London:

Routledge.

Kagan, H. K., \& Kagan, N. I. (1997). Interpersonal process recall: Influencing human interaction. In C. E.Watkins Jr. (Ed.), Handbook of psychotherapy supervision (pp. 296-309). New York: Wiley.

MacDougall, C. (2002) Rogers's Person-Centered Approach: Consideration for Use in Multicultural Counseling, Journal of Humanistic Psychology, 42 (2), 48-65.

Miller, K. E., Omidian, P., Kulkarni, M., Yaqubi, A., \& Rasmussen, A. (2009). The validity and clinical utility of Post-traumatic Stress Disorder in Afghanistan. Transcultural Psychiatry, 46, 219-237.

Ministry of Higher Education Afghanistan (2018) https://www.mohe.gov.af/en/universities (Retrieved in October 2018).

Moracco, J.C. Int J Adv Counselling (1978) 1: 199. https://doi.org/10.1007/BF00120544 (Retrieved in October 2018).

National Mental Health Strategy 2009-2014, Islamic Republic of Afghanistan, Ministry of Public Health General Directorate of Preventive Medicines Mental Health \& DDR Department. Retrieved in September 2018 by:

http://www.mhinnovation.net/sites/default/files/downloads/innovation/research/National-MentalHealth-Strategy.pdf

Page, S. \& Wosket, V. (2003) Supervising the Counsellor: the cyclical model. Taylor \& Francis eLibrary. 
Panter-Brick, C., Eggerman, M., Gonzalez, V., \& Safdar, S. (2009). Violence, suffering, and mental health in Afghanistan: a school-based survey. The Lancet, 374(9692).

Polster, E. and Polster, M. (1973). Gestalt therapy integrated. Vintage Booksn.

Proctor, B. (2008). Group supervision. A guide to creative practice (2nd ed.). London: Sage Publications.

Rogers, C. (1951). Client-centered Therapy: Its Current Practice, Implications and Theory. London: Constable.

Sayed, G.D. (2011) Mental Health in Afghanistan Burden, Challenges and the Way Forward, Health, Nutrition and Population (HNP) discussion paper. World Bank, Washington, DC. (c) World Bank. https://openknowledge.worldbank.org/handle/10986/13589 License: CC BY 3.0 IGO.

Soheilian, S. S., \& Inman, A. G. (2009). Middle Eastern Americans: The effects of stigma on attitudes toward counseling. Journal of Muslim Mental Health, 4, 139-158.

http://dx.doi.org/10.1080/15564900903245766 